\title{
Orbit Verification Results of the De-Orbit Mechanism Demonstration CubeSat FREEDOM
}

\author{
By Hiroki Uto, ${ }^{1)}$ Toshinori KuwahARA, ${ }^{2)}$ and Tomoyuki HondA ${ }^{2)}$ \\ ${ }^{1)}$ Nagoya Technical Center, Nakashimada Engineering Works, Ltd., Kasugai, Japan \\ ${ }^{2)}$ Department of Aerospace Engineering, Tohoku University, Sendai, Japan
}

(Received June 23rd, 2017)

\begin{abstract}
Nakashimada Engineering Works, Ltd. and Tohoku University have developed a 1U-size CubeSat "FREEDOM". The mission of FREEDOM is to demonstrate a thin film deployment mechanism called the De-Orbit Mechanism (DOM) for space debris prevention, and includes deployment of the thin film, early de-orbiting, and tracking of its orbit transition. FREEDOM was released into low Earth orbit from the International Space Station using the robotic arm of the Japanese Experiment Module "Kibo" on January 16, 2017. The initial altitude of FREEDOM was approximately $410 \mathrm{~km}$. The transition of the orbit of FREEDOM was monitored by obtaining the publicly available two line elements. FREEDOM fell below an altitude of $250 \mathrm{~km}$ on February 6, 2017 and is considered to have re-entered into the Earth atmosphere approximately one day later. FREEDOM stayed in orbit approximately 22 days. This result agrees with the numerical estimates and proves that FREEDOM succeeded in the deployment of the film and early de-orbiting.
\end{abstract}

Key Words: De-Orbit Mechanism, Space Debris, CubeSat, International Space Station

\section{Introduction}

Following the increase in the launches of microsatellites, space debris mitigation is a pressing problem. The InterAgency Space Debris Coordination Committee recommends that a satellite's lifetime in Low Earth Orbit (LEO) should be limited up to 25 years. With this recommendation in mind, since 2010 Nakashimada Engineering Works, Ltd. and Tohoku University have been developing the De-Orbit Mechanism (DOM), which deploys a thin film to drag a microsatellite down to re-enter into the Earth atmosphere.

Former models of DOM have already been launched twice as a payload of CubeSat "RAIKO"1) and of Earth observation microsatellite "RISING-2", ${ }^{2)}$ which were developed by Tohoku University. However due to a communication problem, RAIKO was unable to deploy its DOM. RISING-2, on the other hand, is still operational and has not yet activated its DOM. As a miniaturized new model of DOM was developed in 2014, an early space demonstration was indispensable in anticipation of future development and improvement of DOM technologies.

Due to this background, a 1U-size CubeSat "FREEDOM"3), dedicated to the demonstration of the DOM, was developed. The appearance of FREEDOM is shown in Fig. 1. FREEDOM is equipped with the newest model of the DOM and its mission was the demonstration of the deployment of the device in space and the tracking of the resulting orbit transition. The newest DOM is capable of deploying a $1500 \mathrm{~mm}$ square size film, which can de-orbit a $50 \mathrm{~kg}$ microsatellite at an $800 \mathrm{~km}$ altitude within 25 years, according to simulation analysis.

Table 1 summarizes the project schedule of FREEDOM. The development of FREEDOM was started in April 2014 and was finished in December 2015. FREEDOM was handed over to Japan Aerospace Exploration Agency (JAXA) in November
2016 and was integrated into an upgraded JEM Small Satellite Orbital Deployer (J-SSOD), which was developed by JAXA. FREEDOM was transported to International Space Station (ISS) in December 2016 and was released into orbit from Japan Experimental Module "Kibo" on January 16, 2017 (Fig. 2). Six CubeSats including FREEDOM were released on the same day. The details of the satellite release opportunities from Kibo are shown in Ref. 4).

In this paper, the overview and achievements of the project are summarized. This paper describes the mission and system of FREEDOM, its operational method, mission results, and an evaluation of the approach used in this project. Unless otherwise specified, date and time in this paper are written in Japan Standard Time.
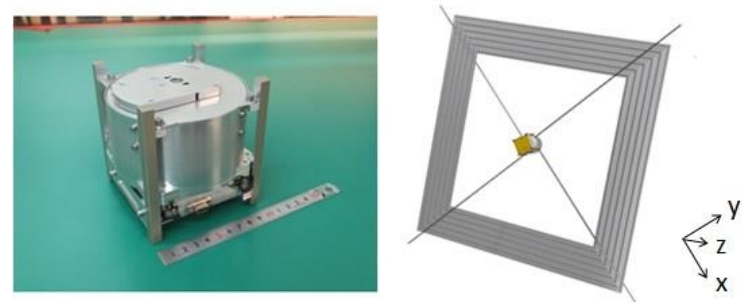

Fig. 1. FREEDOM in launch (left) and deployed (right) configurations.

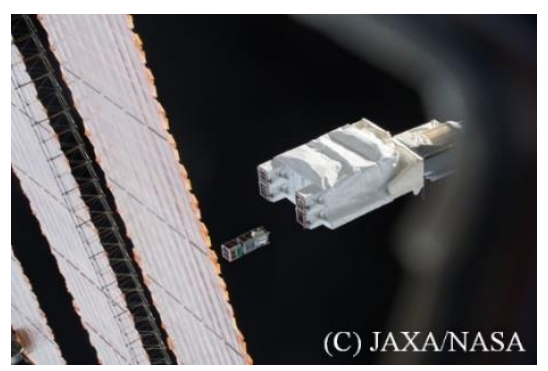

Fig. 2. FREEDOM and two CubeSats released from Kibo. 
Table 1. Chronological table of events of FREEDOM Project.

\begin{tabular}{|c|c|}
\hline Date & Event \\
\hline \multicolumn{2}{|l|}{2014} \\
\hline April & $\begin{array}{l}\text { Started project and development of } \\
\text { Breadboard Model }\end{array}$ \\
\hline April & $\begin{array}{l}\text { Applied for piggyback opportunity } \\
\text { with ASTRO-H }\end{array}$ \\
\hline May & $\begin{array}{l}\text { Applied for release opportunity from } \\
\text { Kibo }\end{array}$ \\
\hline August 27 & $\begin{array}{l}\text { Failed to obtain piggyback } \\
\text { opportunity with ASTRO-H }\end{array}$ \\
\hline September 26 & $\begin{array}{l}\text { Selected as a CubeSat released form } \\
\text { Kibo }\end{array}$ \\
\hline October 8 & $\begin{array}{l}\text { Kickoff meeting } \\
\text { Started to develop Engineering Model } \\
\text { (EM) }\end{array}$ \\
\hline November 18 & Preliminary Design Review \\
\hline October-December & Produced EM Parts \\
\hline \multicolumn{2}{|l|}{2015} \\
\hline January & Assembled EM \\
\hline February 5 & First EM vibration test \\
\hline April 7 & EM fit check \\
\hline April 22 & Second EM vibration test \\
\hline April 27 & Critical Design Review \\
\hline June 17,22 & EM thermal vacuum test \\
\hline June $24-26$ & $\begin{array}{l}\text { EM deployment tests at high and low } \\
\text { temperature }\end{array}$ \\
\hline June 29 & Delta Critical Design Review \\
\hline July 21 & Safety review phase $0 / \mathrm{I} / \mathrm{II}$ \\
\hline July & Started to develop Flight Model (FM) \\
\hline August-September & EM heat cutting test \\
\hline July-September & Produced FM Parts \\
\hline October & Assembled FM \\
\hline November 8-12 & FM thermal vacuum test \\
\hline November 16 & FM fit check \\
\hline November 26 & FM vibration test \\
\hline December 22 & Safety review phase III \\
\hline \multicolumn{2}{|l|}{2016} \\
\hline November 7 & Handed over \\
\hline November 9 & Integration (J-SSOD and FREEDOM) \\
\hline December 9 & $\begin{array}{l}\text { Launched (Kounotori, H-IIB Transfer } \\
\text { Vehicle) }\end{array}$ \\
\hline \multicolumn{2}{|l|}{2017} \\
\hline January 16 & Released into orbit from Kibo \\
\hline February 7 & Re-entered into Earth atmosphere \\
\hline
\end{tabular}

\section{Mission and System}

\subsection{Mission overview}

The intent of the mission of FREEDOM is the demonstration of the DOM1500 device. ${ }^{5)}$ DOM deploys a thin film to drag a microsatellite down to re-enter the atmosphere. DOM1500 is a model number of DOM. The number "1500" represents the size of the film. DOM1500 has a square film with edge lengths of $1500 \mathrm{~mm}$. The material of the film is aluminized polyimide. The appearance of DOM1500 is shown in Fig. 3, however, note that the picture of the deployed configuration is of the Engineering Model (EM) of FREEDOM. A built-in clock timer, which is supplied with electrical power by a primary battery unit, counts 30 minutes from the release into orbit, after which FREEDOM activates DOM1500.

The density of the atmosphere in space is extremely low. The orbit of the satellite decays more quickly when a film is deployed because the atmospheric drag force is increased by the expanded cross-sectional area. On the ground, the operators of FREEDOM obtain orbit information from a public organization and evaluate the de-orbiting performance of the DOM. The mission success criteria are shown in Table 2.

\subsection{System overview}

The system specifications of FREEDOM are summarized in Table 3. FREEDOM is purely intended for DOM demonstration in space and the system is simplified and minimized to the utmost limit. FREEDOM has neither an attitude control system nor a communication system. Commercially available Nickel- Metal Hydride (NiMH) battery cells are used as the battery unit. Though the battery unit is rechargeable, it is not recharged in orbit, as FREEDOM has no solar cells. An electric board called the Satellite Central Unit (SCU) controls the deployment timing of DOM. SCU waits for the defined time period after release into orbit to turn on the power supply to the DOM and to later turn off after DOM deployment. The type of thermal control is passive control. In order to control the satellite temperature in the range in which the satellite can be safely operated, Kapton tape is applied to the surface of the aluminum structure at an appropriate ratio based on thermal analysis. This in because the Kapton tape has a cooling effect that counteracts the heating effect of the surface of aluminum.

\section{Operation Result}

\subsection{Overview of operation}

The Joint Space Operations Center (JSpOC) conducts detection, tracking, and identification of space objects. This information is available on the website "Space-Track". ${ }^{\text {) }}$ The orbital information is provided in the format of Two-Line Element set (TLE).
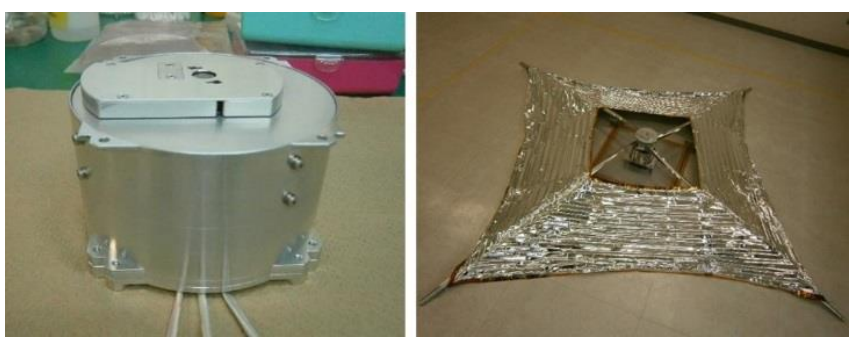

Fig. 3. DOM1500 in stored (left) and deployed (right) configurations.

\begin{tabular}{ll} 
& \multicolumn{1}{c}{ Table 2. Mission success criteria. } \\
\hline Success level & \multicolumn{1}{c}{ Success criteria } \\
Minimum & $\begin{array}{l}\text { Identification of the initial orbit by the information } \\
\text { from a public organization } \\
\text { success }\end{array}$ \\
& $\begin{array}{l}\text { Accumulation of the information of orbit transition for } \\
\text { 1 month }\end{array}$ \\
\hline \multirow{3}{*}{ Full success } & $\begin{array}{l}\text { Accumulation of the information of orbit transition } \\
\text { until the re-entry into the atmosphere of the earth } \\
\text { Evaluation of the de-orbiting performance of DOM } \\
\text { through the comparison with the result of simulation }\end{array}$ \\
\hline \multirow{2}{*}{ Extra success } & $\begin{array}{l}\text { Re-entry to the atmosphere of the Earth within 1 month } \\
\text { Investigation of the effect of weathercock stability on } \\
\text { orbit transition }\end{array}$ \\
\hline
\end{tabular}


Table 3. System Specifications.

\begin{tabular}{ll}
\hline $\begin{array}{l}\text { Size } \\
\text { In storage }\end{array}$ & $\mathrm{W} 112 \times \mathrm{D} 112 \times \mathrm{H} 113.5 \mathrm{~mm}$ \\
After deployment & $\mathrm{W} 1660 \times \mathrm{D} 1660 \times \mathrm{H} 180 \mathrm{~mm}$ \\
\hline Weight & $1.255 \mathrm{~kg}$ \\
\hline Film size & $1500 \times 1500 \mathrm{~mm}$ \\
Outside & $1000 \times 1000 \mathrm{~mm}$ \\
Center cut-off & Circle \\
\hline Orbit & $410 \mathrm{~km}$ \\
Type & $\sim 51.6^{\circ}$ \\
Initial Altitude & About 1 month \\
Inclination & DOM1500 \\
\hline Lifetime & \\
\hline Payload & No attitude control \\
\hline Attitude control & \\
Type & No communication \\
\hline Communication & \\
Type & Passive control \\
\hline Thermal control & Primary battery only \\
Type & NiMH $5 \mathrm{~V}, 2450 \mathrm{mAh}(4$ series of AA battery) \\
\hline Power & \\
Type & \\
Battery & \\
Consumption & \\
\hline
\end{tabular}

Though the Space-Track service requires registration, there are several websites that reprint TLEs and can be browsed without registration. The operators of FREEDOM started obtaining TLEs immediately after FREEDOM was released into orbit. The operators made it a rule to check TLE updates three or four times per day, as the TLEs were updated irregularly and the update frequency was also undefined.

The first TLEs $^{6}$ ) of the six CubeSats released into orbit on January 16, 2017 were opened to the public on January 18, and are listed in Table 4. These CubeSats are ITF-2, WASEDASAT3, FREEDOM, EGG, AOBA-VeloxIII, and TuPOD. The six CubeSats were given the NORAD catalog numbers from 41930 to 41935 , respectively. JSpOC had not identified which satellite corresponded to which number at the time. However, the operators of FREEDOM paid attention to the satellite numbered 41930 , because its $\mathrm{B}^{*}$ term was about 10 times as great as that of the other five CubeSats. $\mathrm{B}^{*}$ term is a quantity which is proportional to cross-sectional area and is inversely proportional to mass. Though $\mathrm{B}^{*}$ term has an aspect to make numerical calculations balance, the large $\mathrm{B}^{*}$ term implied that 41930 was acted on by a large atmospheric drag and had a large cross-sectional area. It was confirmed from the TLEs after release that the altitude of 41930 decreased by $20 \mathrm{~km}$ at a rapid rate. 41930 fell below an altitude of $250 \mathrm{~km}$ on February 6 and is considered to have re-entered the Earth atmosphere approximately one day later.

The TLE of 41930 was updated sixty times for the days during which it stayed in orbit. 41930 was tentatively identified as ITF-2 by JSpOC at the beginning, but was renamed EGG on January 28. It cannot be denied that the operators of FREEDOM had some confusion, because 41930 was identified as EGG when it re-entered. Finally, it was identified as FREEDOM on February 9. The operators were sure of the successful deployment of DOM with this identification.

\subsection{Orbit transition}

The transitions of the altitudes of FREEDOM and ITF-2 were calculated from TLEs and are illustrated in Fig. 4. ITF-2 is a $1 \mathrm{U}$ CubeSat which was released into orbit at the same time together with FREEDOM. ITF-2 has approximately the same mechanical properties as FREEDOM before DOM deployment. Therefore, ITF-2 is an important object as its orbit indicates the reference orbit which FREEDOM would have followed, had DOM not have been deployed.

\subsection{Orbit analysis}

The orbit of FREEDOM with the film deployed is numerically calculated. The orbit initial condition was calculated from the first TLE of FREEDOM on January 17, 2017. The orbit was propagated using the fourth order RungeKutta method based on the gravity field model of an aspherical central body together with the disturbance forces of third-body (sun, moon) perturbations, atmospheric drag, and solarradiation pressure. The method used for the calculation can be seen in Ref. 7) and 8).

Table 4. First TLEs of the six CubeSats released on January 16.

\begin{tabular}{|c|c|c|c|}
\hline \multicolumn{4}{|c|}{0 TBA - TO BE ASSIGNED } \\
\hline $141930 \mathrm{U} 98067 \mathrm{KS}$ & 17017. 95464732 & $.00325713 \quad 00000-0 \quad 44798-20$ & 9998 \\
\hline $241930 \quad 51.6417$ & 67. 23290007822 & 70. $6651289.5001 \quad 15.55584022$ & 132 \\
\hline \multicolumn{4}{|c|}{0 TBA - TO BE ASSIGNED } \\
\hline $141931 \mathrm{U} 98067 \mathrm{KT}$ & 17017. 95489692 & $00016867 \quad 00000-0 \quad 25372-3 \quad 0$ & 9996 \\
\hline $241931 \quad 51.6424$ & 67. 23370009257 & 73. 0121287.156115 .54923161 & 138 \\
\hline \multicolumn{4}{|c|}{0 TBA - TO BE ASSIGNED } \\
\hline $141932 \mathrm{U} 98067 \mathrm{KU}$ & 17017. 95501570 & $.00029840 \quad 00000-0 \quad 44475-3 \quad 0$ & 9997 \\
\hline 24193251.6436 & 67. 23620008109 & 64. 9379295.350015 .54802664 & 137 \\
\hline \multicolumn{4}{|c|}{0 TBA - TO BE ASSIGNED } \\
\hline $141933 \mathrm{U} 98067 \mathrm{KV}$ & 17017. 95502292 & $00038010 \quad 00000-0 \quad 56415-3 \quad 0$ & 9996 \\
\hline $241933 \quad 51.6432$ & 67. 23550008514 & 55. $1435 \quad 305.0255 \quad 15.54790311$ & 132 \\
\hline \multicolumn{4}{|c|}{0 TBA - TO BE ASSIGNED } \\
\hline $141934 \mathrm{U} 98067 \mathrm{KW}$ & 17017. 95504635 & $.00022724 \quad 00000-0 \quad 34123-30$ & 9997 \\
\hline $241934 \quad 51.6403$ & 67. 23510009549 & $\begin{array}{lll}78.9681 & 281.2141 & 15.54747425\end{array}$ & 229 \\
\hline \multicolumn{4}{|c|}{0 TBA - TO BE ASSIGNED } \\
\hline $141935 \mathrm{U} 98067 \mathrm{KX}$ & 17017. 95510838 & $00027590 \quad 00000-0 \quad 41338-3 \quad 0$ & 9992 \\
\hline $241935 \quad 51.6415$ & 67. 23500007001 & 72. 1050 288. 066315.54711190 & 234 \\
\hline
\end{tabular}




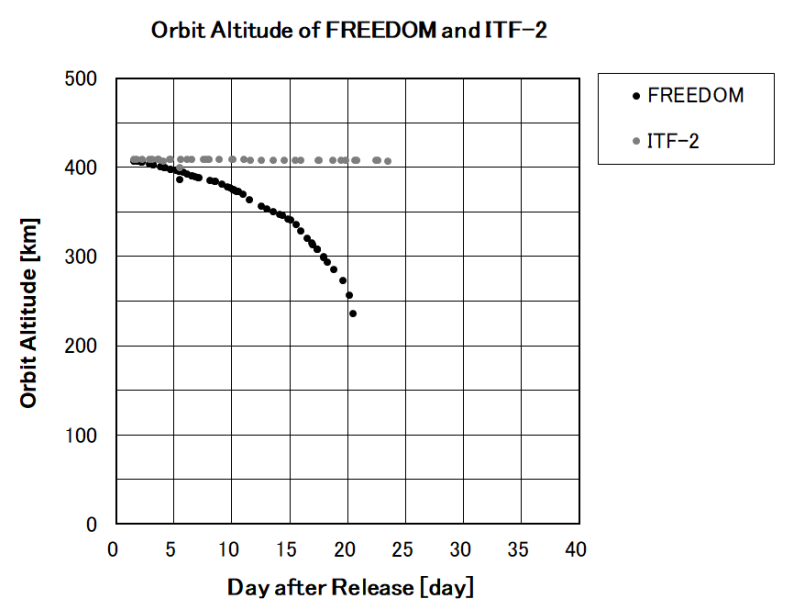

Fig. 4. Orbit altitude of FREEDOM and ITF-2.

The environmental models applied for the simulation are listed in Table 5 and the parameters used are summarized in Table 6. The atmospheric drag coefficients were calculated based on the parameters that were available on January 16, 2017. The cross-sectional areas of $1.25 \mathrm{~m}^{2}, 0.64 \mathrm{~m}^{2}$, and 0.33 $\mathrm{m}^{2}$ correspond respectively to the maximum cross-sectional area, the average cross-sectional area when the film ideally deploys, and the cross-sectional area when the deflection of the film is estimated to be very large. The average cross-sectional area is the value that is the sum of the areas projected on to the $+/-X,+/-Y$, and $+/-Z$ directions divided by 4 . These values are calculated with the outer and inner edge lengths of the film as $1.5 \mathrm{~m}$ and $1.0 \mathrm{~m}$, and the satellite dimension as $0.1 \mathrm{~m}$ cubic, while the effective area of the film in worst case is estimated to be the half of the maximum.

Table 5. Environmental models used in orbital analysis

\begin{tabular}{lll}
\hline Environment & Model & References \\
\hline Earth gravity model & EGM-08, (degree, order) $=(6,0)$ & Ref. 7) \\
Sun/Moon model & DE-200 & Ref. 7), 8) \\
Atmospheric model & NRLMSISE-009) & Ref. 9) \\
\hline
\end{tabular}

Table 6. Parameters used in orbital analysis.

\begin{tabular}{|c|c|c|c|}
\hline Items & Parameters & Value & Reference \\
\hline Atmospheric & $C_{D}$ (drag coefficient) [-] & 2.2 & Ref. 7) \\
\hline \multirow[t]{4}{*}{ drag } & $\mathrm{A}_{\mathrm{p}}$ (daily geomagnetic & 3.0 & Ref. 10) \\
\hline & planetary amplitude) [-] & & \\
\hline & $\begin{array}{l}\mathrm{F}_{10.7} \text { (daily solar flux) } \\
{[\mathrm{SFU}]}\end{array}$ & 78.0 & \\
\hline & $\begin{array}{l}F_{10.7 ~ 81 \text { days }}(81 \text {-day } \\
\left.\text { average of } F_{10.7}\right)[S F U]\end{array}$ & 75.963 & \\
\hline Solar-radiation & $\mathrm{c}_{\mathrm{R}}$ (reflectivity) [-] & 1.0 & Ref. 7) \\
\hline Pressure & $\begin{array}{l}\mathrm{P}_{\text {srp }} \text { (solar-radiation } \\
\text { pressure) }\left[\mathrm{N} / \mathrm{m}^{2}\right]\end{array}$ & 4.57 E-6 & Ref. 7) \\
\hline Time system & $\Delta \mathrm{AT}[\mathrm{s}]$ & 37.0 & Ref. 11) \\
\hline \multirow{5}{*}{$\begin{array}{l}\text { Satellite } \\
\text { Properties }\end{array}$} & Mass of satellite $[\mathrm{kg}]$ & 1.255 & \\
\hline & & & \\
\hline & Cross-sectional area $\left[\mathrm{m}^{2}\right]$ & $1.25(\max )$ & \\
\hline & & 0.64 (avg.), & \\
\hline & & $0.33(\mathrm{~min})$ & \\
\hline
\end{tabular}

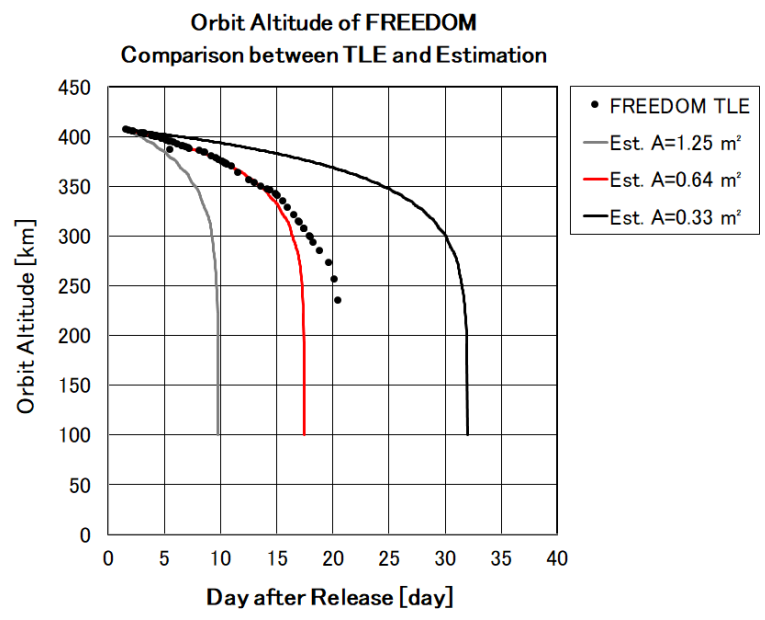

Fig. 5. Result of orbit analysis of FREEDOM.

The results of the analysis are illustrated in Fig. 5. The TLEs of FREEDOM are also plotted in the same figure. According to the results of the analysis, FREEDOM could be expected to stay in orbit for approximately 10 days, 17.5 days, and 32 days when the cross-sectional area is assumed to be $1.25 \mathrm{~m}^{2}, 0.64$ $\mathrm{m}^{2}$, and $0.33 \mathrm{~m}^{2}$ respectively. The lifetime is expected to be more than 1 year if the film is not deployed.

\section{Consideration on Orbit Transition}

\subsection{Comparison of TLEs with analysis}

The values of the TLEs obtained in orbit approximately agree with the simulation results for the average cross-sectional area with the fully deployed film for the first 14 days. Difference between the actual TLEs and simulation appears after 14 days at which point the altitude fell below approximately $350 \mathrm{~km}$. The actual orbital period is longer than the estimation with the average cross-sectional area and the final difference in period was approximately 5 days. It can be estimated that some conditional change might have occurred at around $350 \mathrm{~km}$, such as a change in satellite attitude or in the shape of the film. A detailed investigation of this topic is still being conducted and will be the subject of another publication.

\subsection{Evaluation of de-orbiting capability of DOM}

According to the above discussions, it could be concluded that DOM had successfully deployed in orbit and has accelerated the de-orbiting performance of FREEDOM. The effective area for de-orbiting was about the average crosssectional area, which indicates that the film has been fully deployed and that FREEDOM was freely rotating relative to the velocity vector at the altitude higher than $350 \mathrm{~km}$. Although the lifetime below $350 \mathrm{~km}$ was longer than seen in our analysis, satellites in this altitude de-orbit very rapidly due to the dense atmosphere, and hence it can be concluded that DOM has successfully demonstrated its capability of de-orbiting LEO satellites for space debris prevention.

\subsection{Accomplishment status of mission}

One of the minimum success criteria, "Identification of the initial orbit by the information from a public organization" was accomplished by obtaining TLEs from JSpOC. The other 
criterion, "accumulation of the information of orbit transition for 1 month" ended up a safe side estimation, as the FREEDOM delivered desired de-orbiting performance and de-orbited in only 22 days after deployment. Nevertheless, the orbit of FREEDOM could be tracked continuously throughout the mission, and the objectives of the minimum success criteria could essentially be fulfilled.

"Accumulation of the information of orbit transition until the re-entry into the atmosphere of the earth," was successfully accomplished and is categorized as a full success. "Evaluation of the de-orbiting performance of DOM through the comparison with the result of simulation" was accomplished as illustrated in the analysis result shown in Section 3.3 and the discussions in Sections 4.1 and 4.2.

Regarding the extra successes, "re-entry into the atmosphere of the Earth within 1 month" was accomplished. A detailed analysis on the "investigation of the effect of weathercock stability on orbit transition" is still being conducted and will be the subject of another publication.

\section{Uncertainties in Early Developmental Stage}

\subsection{Operation at low temperature}

Since the DOM's deployment is triggered by the heating of melting wires, it is reasonable to be influenced by a low environmental temperature. NiMH batteries are known to degrade at low temperature, and therefore low temperature environmental tests on the heaters and the NiMH batteries were conducted at $-20{ }^{\circ} \mathrm{C}$, which was the lowest temperature expected in the mission of FREEDOM. It indeed revealed that the battery voltage drops considerably at $-20{ }^{\circ} \mathrm{C}$, in the way that the wire cutting process could take a long period of time. Therefore, SCU was programed to execute the first activation of DOM in 30 minutes after the release for 2 minutes, and the following periodical activations in 170 minutes intervals. Thermal design of the FREEDOM ensured that the temperature of the FREEDOM will be warmed up by the time of second activation, even if the satellite is released at the lowest temperature. Also FREEDOM was equipped with two heaters for redundancy.

\subsection{Size and area of film}

Originally the definition of the film edge length had a range of $1.0 \mathrm{~m}$ to $1.5 \mathrm{~m}$ when the project started, with $1.5 \mathrm{~m}$ as the desired value. Through the development and miniaturization of DOM technologies, EM successfully confirmed that the maximum desired edge length could be achieved. However, pre-flight orbit simulation results indicated that FREEDOM would de-orbit within a few days, too quick to obtain enough orbit transition data for evaluation. Therefore, a decision was made to enlarge the center cut-off of the film of the FM so that the lifetime of the satellite would be extended to about 1 month. The rest of the mechanical specification was kept identical to the EM. Reducing the outer film edge length would have caused a major mechanical design change of the a DOM1500. The standard cut-off of DOM1500 is a $500 \mathrm{~mm}$ square.

\subsection{Action of newly developed mechanism}

The DOM1500 developed for FREEDOM was the smallest and most condensed mechanism among the similar film size ones previously developed by the research group. Deployment tests were conducted before and after vibration tests and it was confirmed that DOM could withstand the vibration conditions of the launch. The third deployment switch of the satellite installed in the side of a rail had few precedent. The secure action of this switch could be confirmed through interface verification and fit check on ground.

\subsection{Area of Kapton tape surface}

Kapton tape was applied to the $+\mathrm{Y},-\mathrm{Y}$, and $-\mathrm{Z}$ surfaces as illustrated in Fig. 6. This is based on the ground simulation results and will ensure that the temperature of FREEDOM will be stabilized in a range from +10 to $+30{ }^{\circ} \mathrm{C}$ after several orbits independent of the initial temperature conditions.

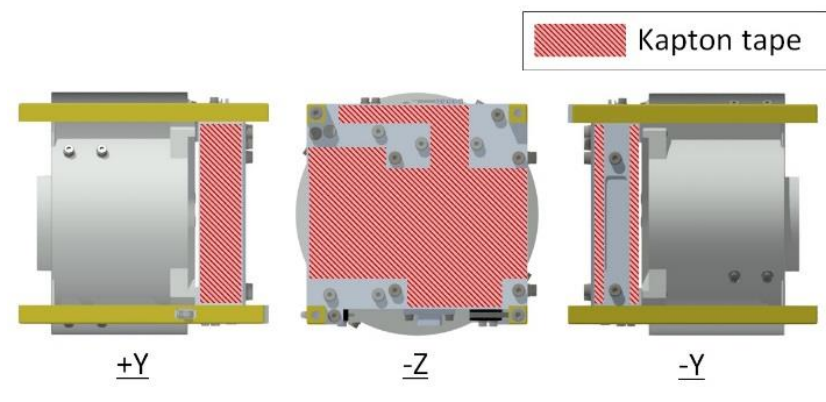

Fig. 6. Area covered by Kapton tape.

\section{Approaches Used for Demonstration}

\subsection{Specialization for DOM demonstration}

Though RISNG-2 was equipped with DOM1500 and was launched in May 2014, it is still operational and has not yet activated its DOM. FREEDOM started to be developed in April 2014 and demonstrated DOM earlier than RISING-2. Therefore, the development of a satellite particularly for DOM is considered to be very effective on early demonstration. Demonstration with RISING-2, on the other hand, will demonstrate that DOM can be activated after storage in a space environment for an extended period of time.

\subsection{Activation of DOM by built-in timer}

By implementing a built-in timer, FREEDOM could eliminate any potential communication problems with the ground station and could successfully deploy DOM. It can be considered the most appropriate and purpose-oriented minimalistic approach to achieve the mission objectives.

\subsection{Tracking by TLE from public organization}

Obtaining the publicly available TLE information was the only way to track the orbit of the FREEDOM as it didn't have communication system. Thankfully FREEDOM could obtain the data, but this was a single point of failure of the mission. It is also true that the identification of the FREEDOM among the other CubeSats released on the same day had a difficulty because FREEDOM was not equipped with a transmitter.

\subsection{Utilization of $1 \mathrm{U}$ CubeSat}

Developing FREEDOM as a $1 \mathrm{U}$ CubeSat imposed severe restrictions on mass and envelope. However, the restrictions also refined the design of DOM. DOM1500 for FREEDOM is approximately $930 \mathrm{~g}$ in weight and $110 \mathrm{~mm}$ in diameter, which is the most compact and lightest design. It was a very unique and rapid demonstration opportunity for DOM. 


\subsection{Extremely simplified system}

The basic policy in designing FREEDOM was to make the satellite system as simple as possible. The following specifications were applied.

- No communication system

- No attitude control system

- No solar cell

- Only primary battery

- Passive thermal control system

These specifications enabled rapid development by a small team of five people. There were no particular problems caused by the absence of a communication system nor an attitude control system. Primary battery and passive thermal control approach also caused no trouble for the project.

\subsection{Release opportunity from ISS}

The ISS release opportunity provided mild launch conditions and frequent access to space. The orbit altitude of around $400 \mathrm{~km}$ was sufficient for the DOM demonstration, but a deorbit demonstration from an even higher orbit will be definitely of interest for DOM.

\section{Conclusion}

This paper summarized the orbit verification results of the CubeSat FREEDOM, which was released into space from Japan Experimental Module "Kibo" of ISS on January 16, 2017. By comparing the observed value of orbit transition measurements with those found in simulation, it can be concluded that FREEDOM succeeded in deploying a thin film and in early de-orbiting. Thus, DOM demonstrated the desired capability as a de-orbit device for active space debris prevention.

Several specifications such as the film size, the area of Kapton tape surface, and the heater-activation sequence, could be determined through analysis, tests, and production.

Though the identification of the FREEDOM orbit using publicly available TLE information was not straightforward, the project approach taken for the early demonstration of the DOM technology onboard the CubeSat FREEDOM was overall a great success.

\section{Future Outlook}

In addition to DOM1500 that was demonstrated this time, DOM500, which has a $500 \mathrm{~mm}$ square film, and DOM2500, which has a $2500 \mathrm{~mm}$ square film, have already been developed. It is easy to install DOM onto a satellite because DOM observes the simplification of the mechanical and electrical interfaces. DOM1500 and DOM2500 are devices for 50kg-class microsatellites. In particular, DOM1500 is designed to be able to be mounted inside a rocket interface cylinders and is likely to be put into practical standardized utilization. The demand for DOM500 is expected to grow with the increasing number of CubeSats. Moreover, DOM4500 for microsatellites that have larger mass or orbiting in higher altitude is currently under development.
One of the future technical challenges is the demonstration of the secure functionality of DOM after a long-term storage period in a space environment for several years. Another challenge is to assess the additional debris risk caused by the increased cross-sectional area of the DOM while de-orbiting. These research topics will be continued so that the DOM can be widely utilized for maintaining future safe space utilization.

\section{Acknowledgments}

The accomplishment described in this paper was made possible by using a release opportunity from Kibo of ISS. Only Kibo, which features unique systems such as the airlock and the robot arm, can provide an opportunity like this. The authors are very grateful to JAXA and the party concerned with the gratis opportunity for demonstration in space. The authors are very grateful to the EGG team, the ITF-2 team, and the WASEDASAT3 team for providing information on their satellites. The authors are also very grateful to JSpOC for tracking space objects, allowing public access to orbital information every day, and for permitting the redistribution of said orbital information.

\section{References}

1) Sakamoto, Y., Tanabe, Y., Yagisawa, H., Sugimura, N., Yoshida, K., Nishio, M., Nakajo, T. and Akiyama, H.: Operation Results of Cubesat RAIKO Released from International Space Station, Trans. JSASS, Aerospace Tech. Japan, 12, ists29 (2014), pp.Tf_7-Tf_12.

2) Kuwahara, T., Sakamoto, Y., Yoshida, K., Takahashi, Y., Fukuhara, T. and Kurihara, J.: Mission and System of the Earth Observation Microsatellite RISING-2, Proceedings of 8th Symposium on Small Satellites for Earth Observation, Berlin, April 4-8, 2011.

3) Mogi, T., Kuwahara, T. and Uto, H.: Structural Design of De-orbit Mechanism Demonstration CubeSat FREEDOM, Trans. JSASS, Aerospace Tech. Japan, 14, ists30 (2016), pp.Pf_61-Pf_68.

4) Akagi, H., Takata, M., Watanabe, H. and Oikawa, K.: Kibo's contribution to broadening the possibilities for Micro/Nano-satellite, Proceedings of the SpaceOps 2016 Conference, Daejeon, Korea, May 16-20, 2016, AIAA 2016-2517.

5) Uto, H., Kuwahara, T. and Mogi, T.: Specifications of Thin Film Deployment Type De-orbit Device DOM1500, 4th UNISEC-Global Meeting, Deorbit Device Competition, Varna, Bulgaria, 2016.

6) Joint Space Operations Center, https://www.space-track.org/ (accessed January 18, 2017).

7) Vallado, D. A. and McClain, W. D.: Fundamentals of Astrodynamics and Applications Fourth Edition, Microcosm Press, Portland, 2013, pp.517-729.

8) Montenbruck, O. and Gill, E.: Satellite Orbits, Springer, 2005, pp.53-116.

9) Picone, J. M., Hedin, A. E. and Drob, D. P.: NRLMSISE-00 empirical model of the atmosphere: Statistical comparisons and scientific issues, Journal of Geophysical Research:Space Physics, 107, SIA 15-1-SIA 15-16, 2002.

10) National Oceanic and Atmospheric Administration, http://www.noaa.gov/ (accessed February 16, 2017).

11) National Geospatial-Intelligence Agency Office of Geomatics, http://earth-info.nga.mil/GandG/update/index.php?action=home (accessed February 16, 2017).

12) International Earth Rotation and Reference Systems Service, https://www.iers.org/IERS/EN/Home/home_node.html (accessed February 16, 2017). 